\title{
Anuário do Instituto de Geociências - UFRJ \\ www.anuario.igeo.ufrj.br
}

\section{Previsão de Nevoeiro Utilizando Multicritérios Baseados em Simulações do Modelo WRF para o Aeroporto Internacional Afonso Pena Multirule Fog Forecast Based on WRF Simulations for Afonso Pena International Airport}

\author{
José Eduardo Gonçalves Platenik ${ }^{1}$; Gutemberg Borges França²; Antonio Vicente \\ Pereira Neto ${ }^{1}$; Ricardo Marcelo da Silva² \&Vinícius Albuquerque de Almeida ${ }^{2}$
}

\author{
${ }^{1}$ Centro Integrado de Meteorologia Aeronáutica (CIMAER), Departamento de Controle do Espaço Aéreo \\ Brasileiro (DECEA), Ponta do Galeão, s/no, Ilha do Governador, 21941-520, Rio de Janeiro, RJ, Brasil. \\ ${ }^{2}$ Laboratório de Meteorologia Aplicada, Departamento de Meteorologia-IGEO-CCMN, Universidade Federal do Rio de Janeiro \\ (UFRJ), Av. Athos da Silveira Ramos,274, 21941-916, Cidade Universitária, Ilha do Fundão, Rio de Janeiro, RJ, Brasil \\ E-mails: platenikjegp@fab.mil.br; gutemberg@lma.ufrj.br; pereiraavpn@fab.mil.br; \\ ricardomarcelo@lma.ufrj.br; vinicius@lma.ufrj.br \\ Recebido em: 07/07/2020 Aprovado em: 30/09/2020 \\ DOI: http://dx.doi.org/10.11137/2020_4_376_383
}

\section{Resumo}

Desenvolve-se um método para previsão de nevoeiro no Aeroporto Internacional Afonso Pena, Paraná - Brasil. Utiliza-se uma abordagem de multicritérios ajustados com registros de dados meteorológicos observados e previstos, a cada 15 minutos, pela estação meteorológica de superfície e dados simulados pelo modelo numérico Weather Research and Forecasting, respectivamente, considerando 30 dias de ocorrência de eventos de nevoeiro no período entre maio e julho de 2019. As correlações entre os dados, previstos e observados, de temperatura, umidade relativa e velocidade do vento são iguais a 0,93, 0,89 e 0,74, respectivamente. Quatro critérios foram estabelecidos via combinação das variáveis meteorológicas mencionadas, isto é: (i) umidade relativa e temperatura; (ii) umidade relativa e temperatura do ar; (iii) umidade relativa, temperatura do ar e velocidade do vento; e (iv) umidade relativa, temperatura do ar, velocidade e direção do vento. Estes foram testados e as probabilidades de acerto para os critérios I, II, III e IV, da previsão do evento, para início (término) são, respectivamente, de 93,2\% (91,5\%), 93,2\% (93,7\%), 88,1\% (88,7\%) e 90\% (87,5\%). As previsões possuem uma leve tendência a atrasar o início e adiantar o término do nevoeiro.

Palavras-chave: Nevoeiro; Multicritérios; Previsão

\section{Abstract}

It develops a method for fog forecast at Afonso Pena International Airport, Paraná - Brazil. A multirule approach is used, based on 15-minute observed and predicted meteorological data, from the automatic weather surface station and simulated data using the numerical model Weather Research and Forecasting, respectively, considering 30 days of fog events during May-July 2019. The correlation for the predicted and observed temperature, relative humidity, and wind speed data are equal to 0.93 , 0.89, and 0.74 , respectively. The application of the four established multirule indicates that the probability of detection by rule (I, II, III, and IV) of the event forecast for the onset (demise) are, respectively, 93.2\% (91.5\%), 93.2\% (93.7\%), 88.14\% (88.7\%), and 90\% (87.5\%). The fog forecasts are slightly biased, i.e., delayed onset and anticipated demise in 30 minutes or less.

Keywords: Fog; Multirule; Forecast 


\section{Introdução}

Em geral, eventos meteorológicos com restrição de visibilidade (por exemplo: nevoeiro, precipitação e outros) são de grande impacto para as operações de pouso e decolagem nos aeroportos. Gultepe et al. (2017a,b) apresentam dados que demonstram as expressivas perdas financeiras da ordem de US\$ 50 milhões devido a três dias de nevoeiro no Reino Unido.

Em particular o nevoeiro, definido pela Organização Meteorológica Mundial (World Meteorological Organization -WMO) como a suspensão na atmosfera de gotículas d'água, ou cristais de gelo, reduzindo a visibilidade horizontal a um valor menor que 1.000 (mil) metros (WMO, 1992), impacta as operações de pouso e decolagem nos aeroportos brasileiros (INFRAERO, 2019). É comum classificar os nevoeiros como: de radiação (causado pelo resfriamento da superfície terrestre por perda radiativa, tipicamente em noites sem nuvem), e de advecção (causada pelo deslocamento de ar quente e úmido sobre uma superfície mais fria). Os trabalhos de Oke (1988), Varejão-Silva (2006), França et al. (2018), entre outros, detalham os mecanismos de formação e de classificação dos nevoeiros.

Na aviação, a previsão dos fenômenos meteorológicos é essencial para o planejamento, a execução da navegação aérea e o gerenciamento de fluxo aéreo, visando, não apenas a segurança do voo, mas também a otimização dos recursos e a logística empregada nesta área. As perdas totais associadas ao impacto do nevoeiro nos transportes aéreos são de extrema preocupação para o setor aeronáutico, necessitando de medidas de segurança específica para prevenir acidentes, atrasos ou cancelamentos dos voos (Gultepe et al., 2019).

Uma das ferramentas mais utilizadas atualmente nos serviços operacionais de previsão meteorológica são os modelos numéricos, que são baseados em equações diferenciais parciais para gerar prognósticos, que descrevem a evolução espaço-temporal de diversas grandezas meteorológicas (Skamarock et al., 2019). No entanto, apesar dos avanços no desenvolvimento de modelos atmosféricos, estes ainda necessitam ser aprimorados para vencer o desafio da previsão das condições para variação de visibilidade (incluindo nevoeiro) de forma assertiva e fidedigna como requer à aviação (Gultepe, 2006; Croft \& Ward, 2015).

Gultepe et al. (2019) fez uma revisão do conhecimento disponível em meteorologia para operações aeronáuticas e incluiu nesta revisão uma descrição do ambiente propício da camada limite para a ocorrência de nevoeiro, analisando as variáveis geradas por um modelo atmosférico regional.

França et al. (2018) investigaram dois eventos de nevoeiro no Aeroporto Internacional de Guarulhos, São Paulo - Brasil, usando dados coletados em alta frequência (15 minutos) de estações de superfície automáticas e do perfil vertical de vento da baixa troposfera extraídos pelo Sound Detection And Ranging (SODAR) e a radiossondagem, a cada 12h, do Aeroporto Campo de Marte, São Paulo-Brasil, mostrando que os processos associados a fenômenos de baixa visibilidade (início, duração e fim) estão correlacionados com o comportamento da camada limite (ex: altura da camada, energia cinética turbulenta, intensidade do vento, entre outros).

Payra \& Mohan (2014), por sua vez, desenvolveram um procedimento que apresenta resultados de previsão de nevoeiro bastante assertivos - com 24 horas de antecedência - baseado no ajuste de uma árvore de decisão (multicritério), considerando dados meteorológicos de superfície de Nova Delhi, na Índia, para caracterização de eventos de nevoeiro, e dados prognosticados gerados pelo modelo atmosférico Weather Research and Forecasting (WRF, Skamarock et $a l ., 2019)$. Os resultados de ocorrência de nevoeiro e nãonevoeiro apontam uma taxa de acerto de cerca de $94 \%$ e com acurácia do início dos eventos variando de 30 a 90 minutos.

No Brasil, o Departamento de Controle do Espaço Aéreo (DECEA) é o setor responsável por prover os meios necessários (sistemas e equipamentos) para o gerenciamento, o controle e a segurança do serviço de navegação aérea, de modo seguro e eficiente, conforme estabelecido nas normas nacionais e nos acordos e tratados internacionais, estabelecidos pela Organização de Aviação Civil Internacional (OACI), da qual o Brasil é signatário (Decreto Federal $n^{\circ} 21.713 / 1946$, Lei Complementar $n^{\circ}$ 97/1999 e Decreto Federal n 6.835/2009). Para cumprir o estabelecido pela OACI, o DECEA mantém um serviço de meteorologia aeronáutica, com emissão de previsões meteorológicas rotineiras, que incluem a previsão de visibilidade horizontal reduzida, quer seja com enfoque de segurança de voo, quer seja com enfoque no gerenciamento de fluxo de tráfego aéreo, sendo esta considerada uma informação essencial para otimizar recursos, contribuindo com a segurança e eficiência dos voos.

A região do Aeroporto Internacional Afonso Pena, Paraná - Brasil, - área deste estudo - é uma das mais impactadas devido às restrições impostas pelos eventos de baixa visibilidade. Esse aeroporto é o sexto maior do país em movimentação de aeronaves, quarto em movimento de passageiros, com cerca de 210 (duzentos e dez) pousos e decolagens diárias, e tem suas operações de pouso e decolagem impactadas em aproximadamente 300 (trezentas) horas por ano (INFRAERO, 2019; Oliveira, 2019). Dessa forma, devido ao atual grau de subjetividade utilizado pelo meteorologista para elaborar previsão de nevoeiro para essa localidade, faz-se necessário o desenvolvimento de modelos prognósticos objetivos.

Pereira (2014) desenvolveu uma primeira abordagem preditiva de nevoeiro baseado em redes neurais artificiais, 
para a mesma área de estudo deste trabalho, e seus resultados apontam altos valores de razão de falso alarme, aproximadamente $96 \%$, superestimando significativamente (em seis vezes) a ocorrência do fenômeno.

O objetivo deste trabalho é desenvolver um modelo preditivo, de início e término dos eventos de nevoeiro, com uma antecedência de 24 horas, para o Aeroporto Internacional Afonso Pena, Paraná - Brasil, utilizando a abordagem de multicritérios (árvore de decisão), baseado no trabalho de Payra \& Mohan (2014).

\section{2 Área de estudos e dados}

Na Figura 1 é apresentado o domínio utilizado nas simulações do WRF, detalhado na seção 4, com as grades aninhadas de resolução espacial de 9,3 e $1 \mathrm{~km}$, centrada no Aeroporto Internacional Afonso Pena, São José dos Pinhais (ponto preto), que está localizado na latitude $25^{\circ}$ $32^{\prime}$ 'S e longitude $49^{\circ} 10^{\prime} \mathrm{W}$.

Na Tabela 1 são apresentados os detalhes (fonte, frequência e variáveis meteorológicas) dos dados utilizados

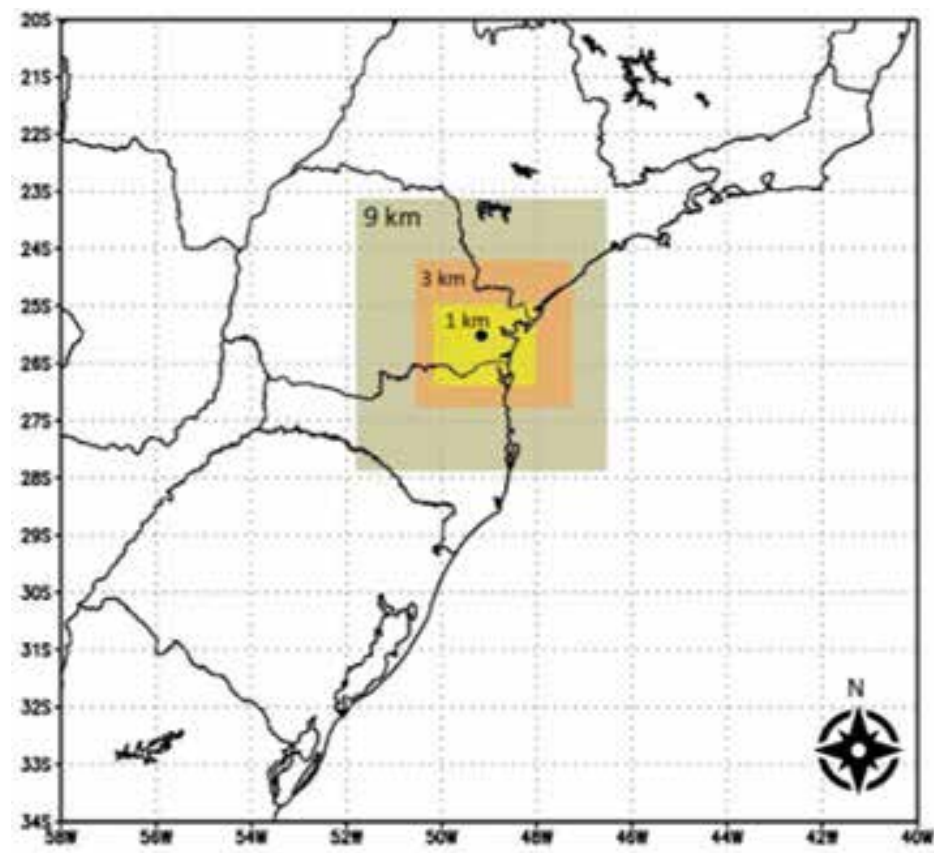

Figura 1 Domínio computacional, com grades aninhadas de 9, 3 e $1 \mathrm{~km}$, para as simulações com o WRF. O ponto preto representa a localização aproximada do Aeroporto Internacional Afonso Pena.

\begin{tabular}{|c|c|c|c|}
\hline Fonte & Período & Frequência & Variáveis \\
\hline $\begin{array}{l}\text { Estação Meteorológica de Superfície } \\
\text { (EMS) automática1 }\end{array}$ & 2019 & 15 minutos & $\begin{array}{l}\text { pressão atmosférica }(\mathrm{hPa}) \text {, temperatura do ar }\left({ }^{\circ} \mathrm{C}\right) \text {; Temperatura do bulbo úmido } \\
\left({ }^{\circ} \mathrm{C}\right) \text {, umidade relativa do ar }(\%) \text {; precipitação }(\mathrm{mm}) \text {; radiação solar }\left(\mathrm{W} / \mathrm{m}^{2}\right) \text {; } \\
\text { Alcance Visual da Pista }(\mathrm{RVR}-\text { Runway Visual Range })(\mathrm{m}) \text {; direção do vento } \\
\left({ }^{\circ}\right) \text { e velocidade do vento }(\mathrm{m} / \mathrm{s}) \text {. }\end{array}$ \\
\hline $\begin{array}{l}\text { Meteorological Aerodrome } \\
\text { Report (METAR) e observações } \\
\text { especiais (SPECI) }\end{array}$ & 2019 & até $1 \mathrm{~h}$ & $\begin{array}{l}\text { pressão atmosférica }(\mathrm{hPa}) \text {, temperatura do ar }\left({ }^{\circ} \mathrm{C}\right) \text {; Temperatura do bulbo úmido } \\
\left({ }^{\circ} \mathrm{C}\right) \text {, umidade relativa do ar }(\%) \text {; direção do vento }\left({ }^{\circ}\right) \text { e velocidade do vento }(\mathrm{m} / \mathrm{s}) \text {; } \\
\text { cobertura de nuvem; visibilidade horizontal (unidade de comprimento); tempo } \\
\text { presente. }\end{array}$ \\
\hline $\begin{array}{l}\text { Global Forecast System (GFS) } \\
\text { / National Centers for Environmental } \\
\text { Prediction (NCEP) }{ }^{1}\end{array}$ & 2015-presente & $3 \mathrm{~h}$ & Variáveis para inicialização e contorno das simulações com o modelo WRF² \\
\hline \multicolumn{4}{|c|}{$\begin{array}{l}{ }^{1} \mathrm{https}: / / \text { www.redemet.aer.mil.br/ } \\
{ }^{2} \mathrm{https}: / / \text { rda.ucar.edu/datasets/ds084.1/ } \\
{ }^{3} \mathrm{https} / / / \text { rda.ucar.edu/datasets/ds084.1/\#metadata/grib2_levels.html?_do=y }\end{array}$} \\
\hline
\end{tabular}

Tabela 1 Dados utilizados. 
para estudo do comportamento de visibilidade na área de estudo e estabelecimento dos multicritérios prognósticos de nevoeiro.

\section{Método}

Na Figura 2 são apresentados os passos do método utilizado, descritos como segue:

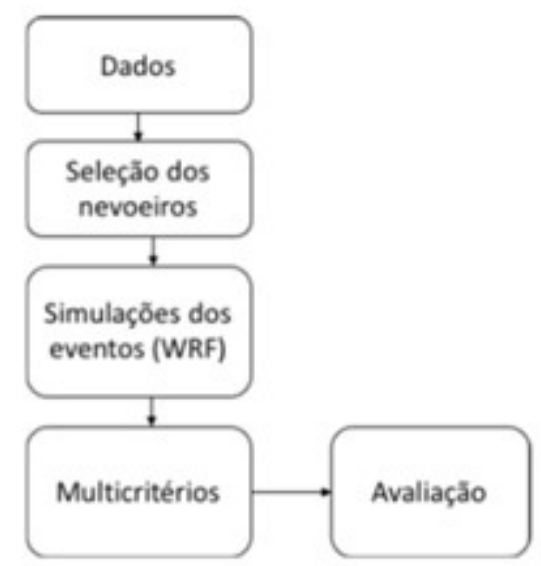

Figura 2 Diagrama de bloco dos passos do método.

Passo 1: Coletam-se os dados das fontes (ver Tabela 1);

Passo 2: Selecionam-se os eventos de nevoeiro, com base na visibilidade do alcance visual da pista (RVR, em inglês);

Passo 3: Simulam-se as condições atmosféricas utilizando o modelo WRF, com saídas a cada 15 minutos, iniciadas à $00 \mathrm{Z}$ do dia anterior aos eventos (selecionados no Passo 2), removendo-se $12 \mathrm{~h}$ para spin-up. As simulações foram executadas utilizando-se dados de previsão (condições iniciais e de contorno) do modelo GFS do NCEP (conforme Tabela 1). Skamarock et al., (2019) apresentam uma descrição abrangente sobre o modelo WRF utilizada como ferramenta de simulação. Diversos autores têm estudado diferentes configurações do modelo WRF para simulação de processos físicos dentro da camada limite (ex: nevoeiro), vide Goswami \& Tyagi (2007), Mohan \& Bhati (2011), Goswami \& Sarkar (2017), Naira et al. (2017) e Pithani et al. (2018). Neste trabalho, adota-se a configuração do WRF utilizada por Payra \& Mohan (2014) - com base nos testes de sensibilidade de Mohan \& Bhati (2011) - isto é:

a) Estabeleceram-se três domínios computacionais centrados, aproximadamente, no aeroporto internacional Afonso Pena (Figura 1) que abrange a área entre $20^{\circ} \mathrm{S}-34^{\circ} \mathrm{S}$ e $40^{\circ} \mathrm{W}-58^{\circ} \mathrm{W}$. As resoluções horizontais aplicadas nos três domínios são de $9 \mathrm{~km}$ (60 x 60 pontos), $3 \mathrm{~km}$ (112 x 97 pontos) e $1 \mathrm{~km}$ (214 x 160 pontos), conforme Figura 1; b) Utiliza-se uma discretização vertical com 33 níveis (conforme Payra \& Mohan, 2014), dos quais 10 níveis encontram-se abaixo de $2.500 \mathrm{~m}$; e

c) Selecionaram-se as parametrizações de processos físicos: Kessler Scheme (Kessler, 1969) para microfísica, Kain-Fritsch Scheme (Kain, 2004) para nuvens do tipo cumulus; Rapid Radiative Transfer Model-RRTMG (Iacono et al., 2008) para radiação de ondas longa e curta; MellorYamada-Janjic Scheme (MYJ) (Janjic, 1994) para camada limite planetária; e Noah-MP Land Surface Model (Niu et al., 2011; Yang et al., 2011) para processos de superfície; Eta Similarity Scheme (Janjic, 1994) para camada de superfície.

Passo 4: Analisa-se o comportamento de uma amostra dos dados observacionais (temperatura, umidade relativa e vento, este último responsável pela homogeneização da camada limite, gatilho para formação de nevoeiro, e a gênese dos nevoeiros de advecção) dos eventos versus os dados simulados pelo WRF (para o ponto de grade mais próximo do aeroporto), conforme o princípio da formação dos nevoeiros como descrito, por exemplo, em Oke (1988), Varejão-Silva (2006) e Payra \& Mohan (2014). Os multicritérios (intervalos do comportamento das variáveis meteorológicas) são estabelecidos para detecção das condições de formação e dissipação do nevoeiro; e

Passo 5: Aplicam-se os multicritérios em uma amostra independente de teste dos eventos de nevoeiro e avalia-se estatisticamente o desempenho usando a correlação e taxa percentual de acerto.

\section{Resultados}

\subsection{Seleção dos nevoeiros}

De acordo com ICEA (2019), ocorreram 3134 registros de nevoeiro no aeroporto de internacional Afonso Pena, correspondendo a mais de 600 horas de nevoeiro por ano, no período de 2007 a 2011. Neste estudo foram utilizados dados de alta frequência da EMS automática, apenas disponíveis a partir de 2019, conforme Tabela 1. Destes últimos, 30 datas foram selecionadas considerando com registro nevoeiro entre maio e julho de 2019. Dentre estes, 8 dias com eventos de nevoeiro (intensos ou não) foram aleatoriamente selecionados para estabelecimento dos multicritérios e os demais para avaliação, conforme Tabela 2 .

\subsection{Simulação do WRF e estabelecimento dos multicritérios}

O modelo atmosférico WRF foi utilizado para simulação (conforme configuração descrita no passo 3 do método) de todos os dias listados na Tabela 2 e os dados 
observados e modelados foram analisados. Observando o comportamento das variáveis temperatura, umidade relativa e velocidade do vento, em intervalos de 15 minutos, as correlações observadas são, respectivamente, 0,93, 0,89 e 0,74. Nas Figuras 3(A-C) são apresentados os comportamentos previstos e observados da temperatura, umidade relativa e vento, para 8 eventos de nevoeiros (conforme Tabela 2, coluna 4) no período de 3 a 5 de maio de 2019, exemplificando a coerência das previsões com as observações, análogo a Payra \& Mohan (2014). Nas Figuras 3(D-E) são apresentados o comportamento da direção e intensidade do vento, observado e previsto, utilizados para ajuste dos multicritérios durante 8 dias de dados, correspondendo a 22 eventos de nevoeiro (ver Tabela 2).

O estabelecimento de critérios é normalmente uma tarefa de tentativa e erro, que consome tempo, até se atingir uma solução ótima entre a detecção do evento e minimização do falso alarme. Tendo como base a concordância do comportamento das variáveis meteorológicas previstas e observadas, foram estabelecidos os seguintes critérios:

I) umidade relativa do ar maior que $95 \%$ e velocidade do vento entre $0,5 \mathrm{~m} / \mathrm{s}$ e $3 \mathrm{~m} / \mathrm{s}$;

II) umidade relativa do ar maior que $95 \%$ e temperatura do ar a $2 \mathrm{~m}$ de altura, entre $8{ }^{\circ} \mathrm{C}$ e $16^{\circ} \mathrm{C}$;

\begin{tabular}{|c|c|c|c|c|}
\hline Dia & Início (h) & Término (h) & Eventos $^{1}$ & Uso \\
\hline $26 / 05 / 19$ & $04: 30$ & $12: 30$ & 3 & \multirow{8}{*}{$\begin{array}{l}\text { Estabelecimento de } \\
\text { Multicritério }\end{array}$} \\
\hline $27 / 05 / 19$ & 01:45 & $10: 45$ & 4 & \\
\hline $04 / 06 / 19$ & $00: 00$ & $10: 00$ & 4 & \\
\hline 07/06/19 & $03: 45$ & $11: 40$ & 2 & \\
\hline $11 / 06 / 19$ & $01: 45$ & $23: 00$ & 3 & \\
\hline $12 / 06 / 19$ & 03:30 & $10: 45$ & 2 & \\
\hline $25 / 07 / 19$ & $04: 30$ & $04: 45$ & 1 & \\
\hline $26 / 07 / 19$ & $06: 30$ & $10: 30$ & 3 & \\
\hline $03 / 05 / 19$ & $00: 45$ & $11: 30$ & 3 & \\
\hline $04 / 05 / 19$ & 07:30 & $10: 45$ & 4 & \\
\hline 05/05/19 & $08: 15$ & $08: 30$ & 1 & \\
\hline $21 / 05 / 19$ & $00: 00$ & $12: 30$ & 3 & \\
\hline $22 / 05 / 19$ & 03:30 & $10: 00$ & 5 & \\
\hline 08/06/19 & $05: 45$ & 09:45 & 3 & \\
\hline $09 / 06 / 19$ & $05: 15$ & $12: 45$ & 2 & \\
\hline $10 / 06 / 19$ & 09:00 & $10: 45$ & 2 & \\
\hline $13 / 06 / 19$ & $04: 45$ & $10: 45$ & 6 & \\
\hline $18 / 06 / 19$ & 04:15 & 07:15 & 1 & \\
\hline $19 / 06 / 19$ & $05: 30$ & 09:45 & 2 & Avaliar̃̃ก \\
\hline $22 / 06 / 19$ & $04: 00$ & $04: 15$ & 1 & Aválilaçau \\
\hline $23 / 06 / 19$ & 04:00 & $13: 00$ & 2 & \\
\hline $24 / 06 / 19$ & $08: 15$ & $08: 30$ & 1 & \\
\hline 09/07/19 & $00: 45$ & $13: 15$ & 2 & \\
\hline $10 / 07 / 19$ & $01: 30$ & $03: 15$ & 1 & \\
\hline $11 / 07 / 19$ & 04:00 & $09: 45$ & 2 & \\
\hline $12 / 07 / 19$ & 04:00 & $11: 00$ & 5 & \\
\hline $20 / 07 / 19$ & $10: 15$ & $12: 30$ & 1 & \\
\hline $24 / 07 / 19$ & 08:00 & $10: 30$ & 3 & \\
\hline $27 / 07 / 19$ & 07:00 & 07:30 & 1 & \\
\hline $31 / 07 / 19$ & $02: 45$ & $00: 00$ & 8 & \\
\hline
\end{tabular}

${ }^{1}$ O número 1 representa evento de nevoeiro (visibilidade $<1000$ metros) contínuo e maior do que 1 representa quantas vezes ao longo do período (início e final) a visibilidade foi inferior a 1000 metros, havendo descontinuidade.

Tabela 2 Datas selecionadas para estabelecimento de multicritérios e avaliação. 


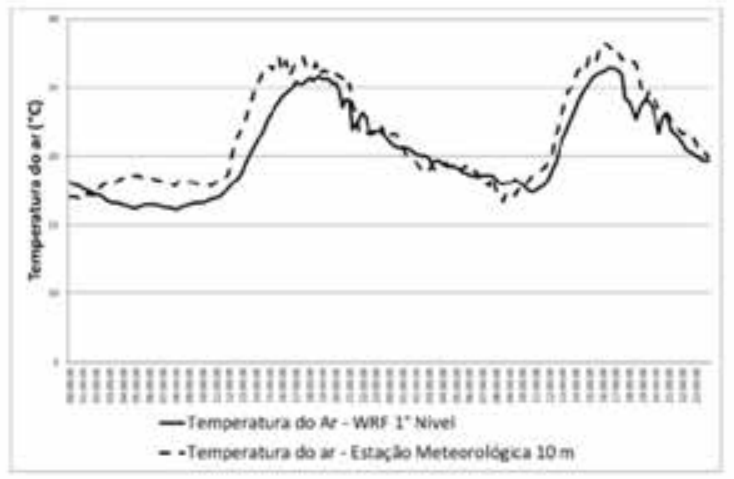

A

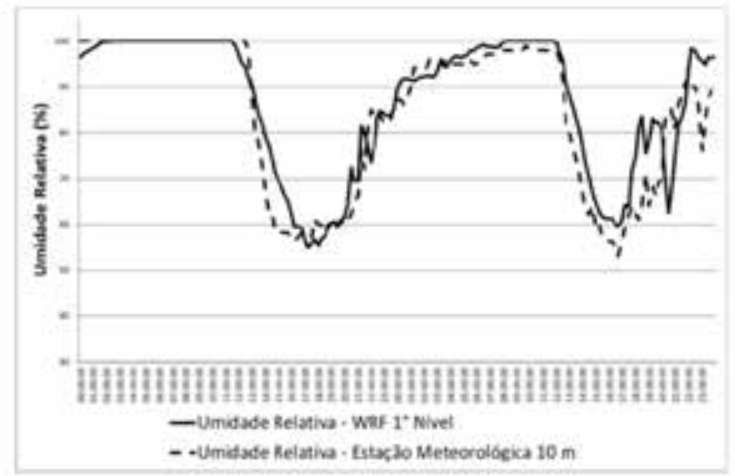

B

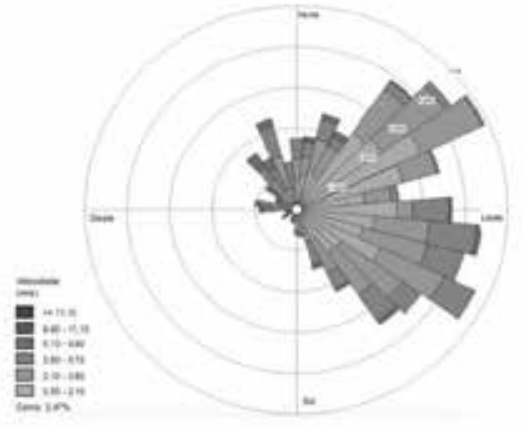

D

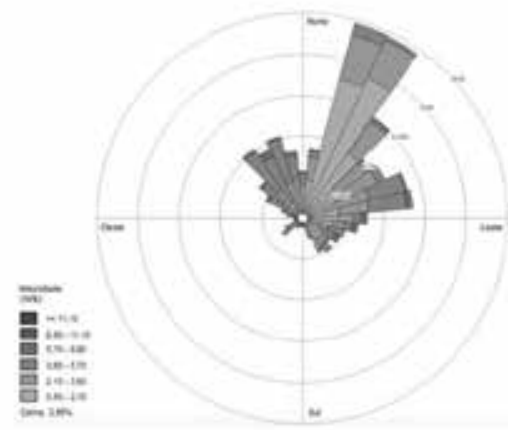

$\mathrm{E}$

Figura 3 Comportamento observado e previsto de: A. temperatura a $2 \mathrm{~m}$, B. umidade relativa do ar a $2 \mathrm{~m}$, C. velocidade do vento para o período entre 3 e 5 de maio de 2019. As Figuras D e E representam a direção-velocidade do vento observado e previsto, respectivamente, para os 8 dias de eventos de nevoeiro utilizados para estabelecimento dos multicritérios.

III) umidade relativa do ar maior que $95 \%$; velocidade do vento entre $0,5 \mathrm{~m} / \mathrm{s}$ e $3 \mathrm{~m} / \mathrm{s}$ e temperatura do ar a $2 \mathrm{~m}$ de altura entre $8{ }^{\circ} \mathrm{C}$ e $16^{\circ} \mathrm{C}$; e
IV) umidade relativa do ar maior que $95 \%$; velocidade do vento entre $0,5 \mathrm{~m} / \mathrm{s}$ e $3 \mathrm{~m} / \mathrm{s}$; temperatura do ar a $2 \mathrm{~m}$ de altura entre $8^{\circ} \mathrm{C}$ e $16^{\circ} \mathrm{C}$; e Direção do vento entre $0^{\circ}$ e $240^{\circ}$. 


\subsection{Aplicação dos multicritérios e avaliação}

A Tabela 3 representa os resultados da aplicação dos quatro critérios conforme definido no passo 4 do método e estabelecidos no item 5.2. Cada linha representa a data, início e término do período de ocorrência dos eventos de nevoeiro (coluna 4), duração total dos eventos no período e as demais colunas representam aplicação dos quatro critérios, ou seja, sim/não) para início (colunas 6 a 9) e término (colunas 10 a 13), respectivamente.

Os resultados mostram que os critérios I e II são ligeiramente superiores aos resultados dos critérios III e IV, individualmente aplicados. Observa-se que o critério
I (relacionado a umidade relativa e velocidade do vento), II (relacionado a umidade relativa e temperatura a $2 \mathrm{~m}$ ), III (relacionado a umidade relativa, velocidade do vento e temperatura a $2 \mathrm{~m}$ ) e IV (relacionado a umidade relativa, velocidade do vento, direção do vento e temperatura a 2 m) são capazes de prever o início (fim) com taxa de acerto de $93,2 \%$ (91,5\%), 93,2\% (93,7\%), 88,1\% (88,7\%) e $90 \%$ $(87,5 \%)$ respectivamente. Os critérios tendem a atrasar o início e adiantar o término dos eventos de 15 a 30 minutos. Em suma, os resultados dos critérios são similares com intervalo de variação do percentual de acerto do início (término) de 88 a $93 \%$ (87 a 93\%).

\begin{tabular}{|c|c|c|c|c|c|c|c|c|c|c|c|c|}
\hline & \multirow{2}{*}{ início (h) } & \multirow{2}{*}{ término (h) } & \multirow{2}{*}{ eventos } & \multirow{2}{*}{ duração (h) } & \multicolumn{4}{|c|}{ Critério de Previsão (início) } & \multicolumn{4}{|c|}{ Critério de Previsão (término) } \\
\hline & & & & & 1 & II & III & IV & 1 & II & III & IV \\
\hline 03/05/19 & $00: 45$ & $11: 30$ & 3 & $04: 30$ & s & s & $\mathrm{n}$ & $\mathrm{n}$ & s & s & $\mathrm{n}$ & $\mathrm{n}$ \\
\hline 04/05/19 & 07:30 & 10:45 & 4 & 01:15 & $\mathrm{n}$ & $n$ & $\mathrm{n}$ & $\mathrm{n}$ & s & s & $\mathrm{n}$ & $\mathrm{n}$ \\
\hline 05/05/19 & 08:15 & 08:30 & 1 & $00: 15$ & $s$ & s & $\mathrm{n}$ & $\mathrm{n}$ & s & s & $\mathrm{n}$ & $\mathrm{n}$ \\
\hline 21/05/19 & 00:00 & $12: 30$ & 3 & $12: 00$ & s & s & s & $s$ & $\mathrm{~s}$ & s & $\mathrm{s}$ & s \\
\hline $22 / 05 / 19$ & 03:30 & $10: 00$ & 5 & $00: 45$ & $\mathrm{~s}$ & $s$ & $\mathrm{~s}$ & $s$ & $\mathrm{~s}$ & $s$ & $\mathrm{~s}$ & $\mathrm{~s}$ \\
\hline 08/06/19 & $05: 45$ & 09:45 & 3 & 02:00 & $\mathrm{s}$ & $\mathrm{s}$ & $\mathrm{s}$ & s & s & $s$ & $\mathrm{n}$ & $n$ \\
\hline 09/06/19 & $05: 15$ & $12: 45$ & 2 & $06: 00$ & $n$ & $n$ & $n$ & $n$ & $n$ & $n$ & $\mathrm{n}$ & $n$ \\
\hline 10/06/19 & 09:00 & 10:45 & 2 & 01:30 & $\mathrm{s}$ & $s$ & $\mathrm{~s}$ & $s$ & $s$ & $\mathrm{~s}$ & $s$ & $\mathrm{~s}$ \\
\hline $13 / 06 / 19$ & $04: 45$ & 10:45 & 6 & $13: 00$ & $s$ & $\mathrm{~s}$ & $\mathrm{~s}$ & $\mathrm{~s}$ & $\mathrm{~s}$ & $\mathrm{~s}$ & $s$ & $\mathrm{~s}$ \\
\hline 18/06/19 & $04: 15$ & 07:15 & 1 & 03:00 & $\mathrm{s}$ & $\mathrm{s}$ & $\mathrm{s}$ & $\mathrm{s}$ & $\mathrm{s}$ & $\mathrm{s}$ & $s$ & $\mathrm{~s}$ \\
\hline $19 / 06 / 19$ & $05: 30$ & 09:45 & 2 & 02:30 & $\mathrm{s}$ & $\mathrm{s}$ & $\mathrm{s}$ & $\mathrm{n}$ & $\mathrm{s}$ & $\mathrm{s}$ & $\mathrm{s}$ & $\mathrm{s}$ \\
\hline $22 / 06 / 19$ & 04:00 & 04:15 & 1 & $00: 15$ & $\mathrm{n}$ & $\mathrm{n}$ & $\mathrm{n}$ & $\mathrm{n}$ & $\mathrm{n}$ & $\mathrm{n}$ & $n$ & $\mathrm{n}$ \\
\hline 23/06/19 & 04:00 & 13:00 & 2 & 08:45 & $\mathrm{s}$ & $\mathrm{s}$ & $\mathrm{s}$ & $\mathrm{s}$ & $\mathrm{n}$ & $\mathrm{n}$ & $\mathrm{n}$ & $\mathrm{n}$ \\
\hline $24 / 06 / 19$ & 08:15 & 08:30 & 1 & $00: 15$ & $\mathrm{~s}$ & $\mathrm{~s}$ & s & $\mathrm{s}$ & $\mathrm{s}$ & $\mathrm{s}$ & $\mathrm{s}$ & $\mathrm{s}$ \\
\hline 09/07/19 & 00:45 & 13:15 & 2 & $12: 00$ & $\mathrm{~s}$ & $\mathrm{~s}$ & $n$ & $n$ & $n$ & $n$ & $\mathrm{n}$ & $\mathrm{n}$ \\
\hline 10/07/19 & $01: 30$ & 03:15 & 1 & $01: 45$ & $\mathrm{~s}$ & $\mathrm{~s}$ & s & $\mathrm{s}$ & $\mathrm{s}$ & $\mathrm{s}$ & $\mathrm{s}$ & $\mathrm{s}$ \\
\hline $11 / 07 / 19$ & 04:00 & 09:45 & 2 & $00: 30$ & $n$ & $n$ & $\mathrm{n}$ & $n$ & $\mathrm{~s}$ & $\mathrm{~s}$ & $n$ & $n$ \\
\hline $12 / 07 / 19$ & 04:00 & 11:00 & 5 & 04:00 & $\mathrm{s}$ & $\mathrm{s}$ & $s$ & $s$ & $n$ & $n$ & $n$ & $n$ \\
\hline 20/07/19 & $10: 15$ & $12: 30$ & 1 & $02: 15$ & $s$ & $\mathrm{~s}$ & $s$ & s & $\mathrm{s}$ & $\mathrm{s}$ & $\mathrm{s}$ & $\mathrm{n}$ \\
\hline $24 / 07 / 19$ & 08:00 & 10:30 & 3 & $00: 45$ & $\mathrm{~s}$ & $\mathrm{~s}$ & $\mathrm{~s}$ & $\mathrm{~s}$ & $\mathrm{~s}$ & $\mathrm{~s}$ & $\mathrm{~s}$ & $\mathrm{~s}$ \\
\hline 27/07/19 & 07:00 & 07:30 & 1 & $00: 30$ & $\mathrm{~s}$ & $\mathrm{~s}$ & $\mathrm{~s}$ & $\mathrm{~s}$ & $\mathrm{~s}$ & $\mathrm{~s}$ & $\mathrm{~s}$ & $\mathrm{~s}$ \\
\hline $31 / 07 / 19$ & $02: 45$ & 00:00 & 8 & 05:00 & $\mathrm{s}$ & $\mathrm{s}$ & $\mathrm{s}$ & $\mathrm{s}$ & $\mathrm{s}$ & $\mathrm{s}$ & $\mathrm{s}$ & $\mathrm{s}$ \\
\hline & & & & Percentual de acerto & $93 \%$ & $93 \%$ & $88 \%$ & $90 \%$ & $91 \%$ & $93 \%$ & $88 \%$ & $87 \%$ \\
\hline
\end{tabular}

Tabela 3 Relação de dias de ocorrência de nevoeiro utilizados para avaliação dos quatro critérios estabelecidos.

\section{Conclusões}

Neste trabalho são estabelecidos multicritérios baseados em dados modelados pelo modelo atmosférico WRF e dados observados - para previsão, de início e término dos eventos de nevoeiro, com simulações iniciadas às $00 \mathrm{Z}$ do dia anterior ao evento, para o Aeroporto Internacional Afonso Pena, Paraná - Brasil.
Os resultados indicam que os quatro critérios estabelecidos são fisicamente representativos da gênese (advecção e radiação) dos eventos de nevoeiro, pois os resultados são similares e capazes de gerar previsões do início e término dos mesmos. No entanto, observa-se que os critérios estabelecidos possuem leve tendência a atrasar o início e adiantar o término do nevoeiro. Como passo futuro, pretende-se estender a amostra de dados analisados, 
visando uma consistência estatística dos resultados, e uso de inteligência computacional (ex: redes neurais e árvore de decisão), visando futura operacionalização do modelo desenvolvido.

\section{Referências}

Croft, P.J. \& Ward B. 2015. Clouds and Fog: Encyclopedia of Atmospheric Sciences. Cambridge, Academic Press, 2998p.

França, g.b.; Carmo, 1.f.r.; Almeida, m.v. \& Neto, f.l.a. 2018. Fog at the Guarulhos International Airport from 1951 to 2015. Journal of Pure and Applied Geophysics, 176: 2191-2202.

Goswami, P. \& Sarkar, S. 2017. An analogue dynamical model for forecasting fog-induced visibility: validation over Delhi. Meteorological Applications, 24: 360-375.

Goswami, P. \& Tyagi, A. 2007. Advance forecasting of onset, duration and hourly fog intensity over Delhi. Research Report RR CM 0714, 27p.

Gultepe, I. 2006. A New Visibility Parameterization for WarmFog Applications in Numerical Weather Prediction Models. Journal of Applied Meteorological and Climatological, 45(11): 1469-1480.

Gultepe, I.; Heymsfield, A.J.; Field, P.R. \& Axisa, D. 2017a. Ice-phase precipitation. Meteorological Monographs, 58: 6.1-6.36.

Gultepe, I.; Heymsfield, A.J.; Gallagher, M.; Ickes, L. \& Baumgardner, D. 2017b. Ice fog: The current state of knowledge and future challenges. Meteorological Monographs, 58: 4.1-4.24.

Gultepe, I.; Sharman, R.; Williams, P.; Zhou, B.; Ellrod, G.; Minnis, P.; Trier, S.; Griffin, S.; Yum, S.S.; Gharabaghi, B.; Feltz, W.; Temimi, M.; Pu, Z.; Storer, L.N.; Kneringer, P.; Weston, M.J.; Chuang, H-y.; Thobois, L.; Dimri, A.P.; Dietz, S.J.; França, G.B.; Almeida, M.V. \& Albuquerque Neto, F.L. 2019. A Review of High Impact Weather for Aviation Meteorology. Journal of Pure and Applied Geophysics, 176: 1869-1921.

Iacono, M.J.; Delamere J.S.; Mlawer, E.; Shepard, M.W.; Clough, S.A. \& Collins, W. 2008. Radiative forcing by long-lived greenhouse gases: Calculations with the AER Radiative transfer models. Journal of Geophysical Research, 113(13): D13103.

ICEA. 2019. Instituto de Controle do Espaço Aéreo, 2019. Sistema de Geração e Disponibilização de Informações Aeronáuticas do Instituto de Controle do Espaço Aéreo. Disponível em $<$ http://clima.icea.gov.br/>. Acesso em 23 de fev. de 2020.

INFRAERO. 2019. Empresa Brasileira de Infraestructura Aeroportuária. Anuário Estatístico Operacional da INFRAERO de 2018. Disponível em <http://www4.infraero.gov.br/ media/677124/ANUARIO_2018.PDF $>$. Acesso em $23 \mathrm{de}$ fev. de 2020 .

Janjic, Z.I. 1994. The step-mountain eta coordinate model: further developments of the convection, viscous sublayer, and turbulence closure schemes. Monthly Weather Review, 122(5): 927-945.
Kain, J.S. 2004. The Kain-Fritsch Convective Parameterization: An Update. Journal of Applied Meteorology, 43(1): 170-181.

Kessler, E. 1969. On the distribution and continuity of water substance in atmospheric circulation. Meteorological Monographs, 10(32): 1-84.

Mohan, M. \& Bhati, S. 2011. Analysis of WRF model performance over subtropical region of Delhi, India. Advances in Meteorology, 2011: 13p.

Naira, C.; Marouane, T.; Michael, W. \& Hosni, G. 2017. Sensitivity of the meteorological model WRF/ARW to planetary boundary layer schemes during fog conditions in a coastal arid region. Atmosheric Research, 187: 106-127.

Niu, G.-Y.; Yang, Z.-L., Mitchell, K.E.; Chen, F.; Ek, M.B.; Barlage, M.; Kumar, A.; Manning, K.; Niyogi, D.; Rosero, E.; Tewari, M. \& Xia, Y. 2011. The community Noah land surface model with multiparameterization options (Noah$\mathrm{mp})$ : 1 . Model description and evaluation with local-scale measurements. Journal of Geophysical Research, 116: D12110.

Oliveira, M.V., 2019. Caracterização e Previsão dos Nevoeiros no Aeródromo SBCT utilizando Inteligência Artificial. Departamento de Meteorologia do Instituto de Geociências, Universidade Federal do Rio de Janeiro, Dissertação de Mestrado, 173p.

Oke, T.R., 1988. The urban energy balance. Progress in Physical Geography, 12: 471-508.

Payra, S. \& Mohan, M. 2014. Multirule Based Diagnostic Approach for the Fog Predictions Using WRF Modelling Tool. Advances in Meteorology, 2014: 11p.

Pereira, M.C.M. 2014. Emprego de Redes Neurais Artificiais RBF na Previsão de Nevoeiro no Aeroporto Internacional Afonso Pena. Programa de Pós-graduação em Métodos Numéricos em Engenharia, Universidade Federal do Paraná, Dissertação de Mestrado, 109p.

Pithani, P.; Ghude, S.D.; Prabhakaran, T.; Karipot, A.; Hazra, A.; Kulkarni, R.; Chowdhuri, S.; Resmi, E.A.; Konwar, M.; Murugavel, P.; Safai, P.D.; Chate, D.M.; Chate, D.M.; Tiwari, Y.; Jenamani, R.K. \& Rajeevan, M. 2018. WRF model sensitivity to choice of PBL and microphysics parameterization for an advection fog event at Barkachha, rural site in the IndoGangetic basin, India. Theoretical and Applied Climatology, 136: 1099-1113.

Skamarock, W.C.; Klemp, J.B.; Dudhia, J; Gill, D.O.; Liu Z.; Berner, J.; Wang, W.; Powers, J.G.; Duda, M.G.; Barker, D.M. \& X.-Y. Huang. 2019. A Description of the Advanced Research WRF Version 4. NCAR Tech. Note, 145p.

Varejão-Silva, M.A. 2006. Meteorologia e Climatologia. Versão digital. Recife, Pernambuco, Brazil, 522p.

WMO. 1992. Vocabulário Meteorológico Internacional. Organização Meteorológica Mundial, Genebra, 802p.

Yang, Y.; Shi, T.; Tang, W.A.; Wu, B.W.; Xun, S.P. \& Zhang, H.Q. 2011. Study of Observational Environment of Meteorological Station Based on Remote Sensing: Cases in six stations of Anhui province. Remote Sensing Technology and Application, 26 (6): 791-797. 